\title{
基于配位辅助的烯烃催化不对称炔氢化
}

\author{
张雯雯王紫璇白小燕李必杰*
}

(清华大学化学系基础分子科学中心 北京 100084)

\begin{abstract}
摘要 烯烃的不对称氢官能团化是一个重要的研究方向. 从简单的烯烃原料出发, 通过该方法可以高效构建手性分子. 多取代烯烃的不对称氢官能团化仍然是一个挑战. 一方面, 烯烃有两个反应位点, 反应的区域选择性需要进行有效的 控制. 另一方面, 如果反应生成了多个手性中心, 则涉及到非对映选择性的控制. 此外, 还需要控制反应的对映选择 性. 因此, 此类研究的关键在于如何发展有效的催化体系, 以同时实现区域选择性、非对映选择性及对映选择性的高效 控制. 针对这一问题, 我们采用配位辅助策略, 利用底物中的配位基团及烯烃与金属中心形成双位点配位模式, 从而 有效控制烯烃转化的区域选择性及立体选择性. 以烯烃不对称炔氢化作为模型转化, 以研究多取代烯烃催化不对称转 化中的选择性控制.
\end{abstract}

关键词＼cjkstart烯烃转化; 不对称催化; 选择性; 配位辅助; 过渡金属催化

\section{Substrate-Directed Catalytic Asymmetric Hydroalkynylation of Alkenes}

\author{
Zhang, Wenwen Wang, Zixuan Bai, Xiaoyan Li, Bijie* \\ (Center of Basic Molecular Science (CBMS), Department of Chemistry, Tsinghua University, Beijing 100084)
}

\begin{abstract}
Catalytic asymmetric hydrofunctionalization of alkene is an important research field, which enables efficient construction of chiral molecules from readily available starting materials. Asymmetric hydrofunctionalization of multiple substituted alkenes represents a significant challenge to organic chemists because this process involves the simutaneous control of regio-, diastereo-, and enantio-selectivities. The key to solve this challenge is to identify novel catalyst systems to exert powerful regio- and stereo-control. Recently, by taking advantage of substrate-directed strategy, we have developed a number of alkene functionalization methods with excellent regio-, diastereo-, and enantio-selectivities. In particular, we focus on catalytic asymmetric hydroalkynylation of alkenes as a model transformation to analyze the factors that control the selectivity. Keywords alkene functionalization; asymmetric catalysis; selectivity; substrate-directed strategy; transition metal catalysis
\end{abstract}

\section{1 研究背景}

烯烃是有机分子中最常见的官能团之一. 烯烃的转 化是有机合成中极为重要的官能团转换过程 ${ }^{[1]}$. 另一方 面, 烯烃是廉价易得的化工原料, 烯烃转化也是化学工 业的基础过程之一. 因此, 不论是在有机合成还是工业 生产中, 烯烃的催化转化长期以来都是非常重要的研究 方向. 高效、实用的烯烃转化方法的发展持续受到化学 家的关注, 新颖的烯烃转化方法的发现可能为有机合成 及化工生产带来变革性影响.

烯烃(特别是内烯烃)的不对称催化转化具有相当的
挑战性 ${ }^{[2]}$. 首先，内烯烃位阻较端位烯烃更大，反应性 能更低. 其次, 烯烃有两个反应位点, 反应的区域选择 性需要得到很好的控制. 再者, 如果反应生成了两个手 性中心，还涉及到非对映选择性的控制. 此外, 对于不 对称的催化转化过程, 还要叠加对映选择性控制的问 题. 因此, 烯烃的区域及立体选择性催化转化是化学家 们长期面临的挑战. 此类研究的关键在于如何开发高效 的过渡金属催化体系，在发展新反应的同时实现区域选 择性、非对映选择性及对映选择性的控制(图 1).

针对这一问题, 我们采用配位辅助策略 ${ }^{[3]}$, 探索烯 烃尤其是内烯烃的不对称催化转化. 配位基团起到多个

\footnotetext{
* Corresponding author. E-mail: bijieli@mail.tsinghua.edu.cn

Received February 16, 2020; revised March 8, 2020; published online March 31, 2020

Project supported by the Thousand Young Talents Program.

青年千人计划资助项目
} 


\section{烯烃区域及立体选择性氢官能团化}

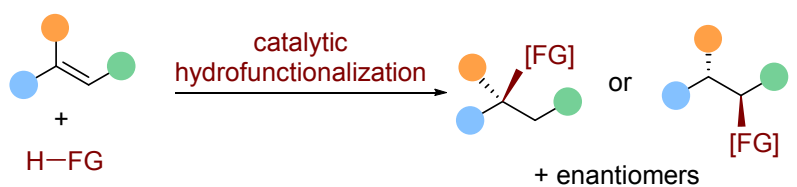

配位辅助的烯烃区域及立体选择性氢官能团化
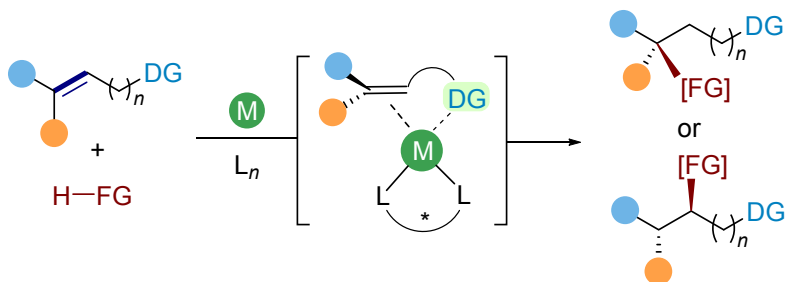

图 1 配位辅助的烯烃不对称转化

Figure 1 Substrate-directed asymmetric alkene functionalization

方面的作用. 首先, 通过金属与配位基团的配位，加快 了底物与催化剂的络合过程, 从而更有利于反应进行. 其次，利用配位基团及烯烃与金属中心形成双位点配位 模式，控制烯烃转化中的选择性包括区域选择性及对映 选择性. 此外, 配位基团本身作为一个官能团存在于反 应产物中, 可以进行后续的转化. 基于此策略, 我们以 烯烃不对称炔氢化作为模型转化研究多取代烯烃、非活 化烯烃不对称催化转化中的选择性控制. 选择炔氢 化 ${ }^{[4,5]}$ 作为研究的切入点基于几个方面的考虑. 一方面, 炔烃同样是有机合成中常见的官能团，原料廉价易得， 基于炔烃的方法学有重要的合成价值 ${ }^{[6]}$. 更为重要的是, 炔氢化产物中的炔烃可以进行多种类型的转化 ${ }^{[7]}$. 例如, 通过氢化、硼氢化、水合、Click 反应等, 炔基能转化为 烷基、烯基、羰基、杂环等官能团. 因此, 构建含有炔 基的手性中心, 再通过后续炔基的转化, 可以合成一系 列含有各种官能团的手性分子砌块(图 2).

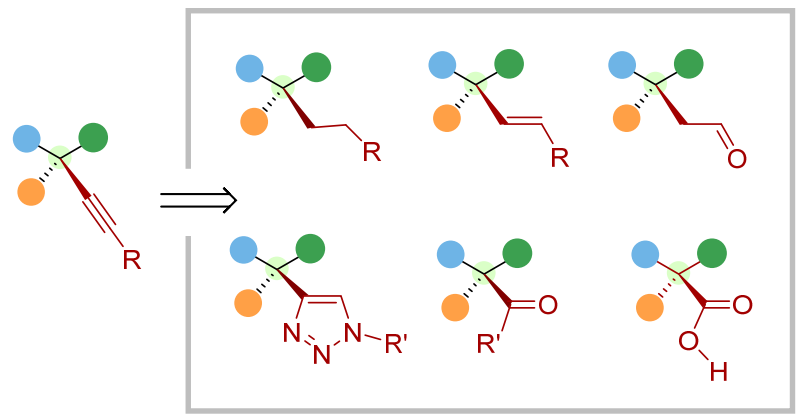

图 2 炔烃的多样性转化

Figure 2 Diverse transformations of an alkyne group

在早期含烯基的醇类化合物的环丙烷化 ${ }^{[8]}$ 及环氧 化 ${ }^{[9]}$ 中, 人们发现羟基通过与金属中心的配位起到了控
制面选择性的作用. 这一策略在后来的烯烃催化氢化中 得到了进一步应用 ${ }^{[10]}$, 很多配位基团包括羟基、羧酸、 酰胺等官能团都可以与金属中心配位，从而控制烯烃氢 化中的面选择性 ${ }^{[11]}$. 以此为基础，陆续发展了配位辅助 的烯烃硼氢化 ${ }^{[12]}$ 、硅氢化 ${ }^{[13]}$ 、酰基氢化 ${ }^{[14]}$ 等反应. 与此 相比, 配位辅助的烯烃不对称催化转化发展较为缓慢, 该类反应主要集中在烯烃不对称氢化. 配位辅助的烯烃 不对称氢官能团化有待进一步发展. 除了配位辅助的烯 烃官能团化以外，配位辅助的碳氢键官能团化领域也得 到了广泛关注 ${ }^{[15]}$.

通过配位辅助策略，我们研究了过渡金属催化的烯 烃不对称氢官能团化过程，发展了多取代及非活化烯烃 的区域及立体选择性炔氢化，构建单个、多个手性中心 包括季碳手性中心，实现了烯烃催化转化中区域选择 性、非对映选择性及对映选择性的高效控制. 此外，通 过实验和计算相结合的方法, 研究了配位辅助的烯烃不 对称炔氢化反应机理，揭示了该类反应的区域及立体选 择性决定因素，探索了反应区域及立体选择性与配体间 的相互关系(图 3).

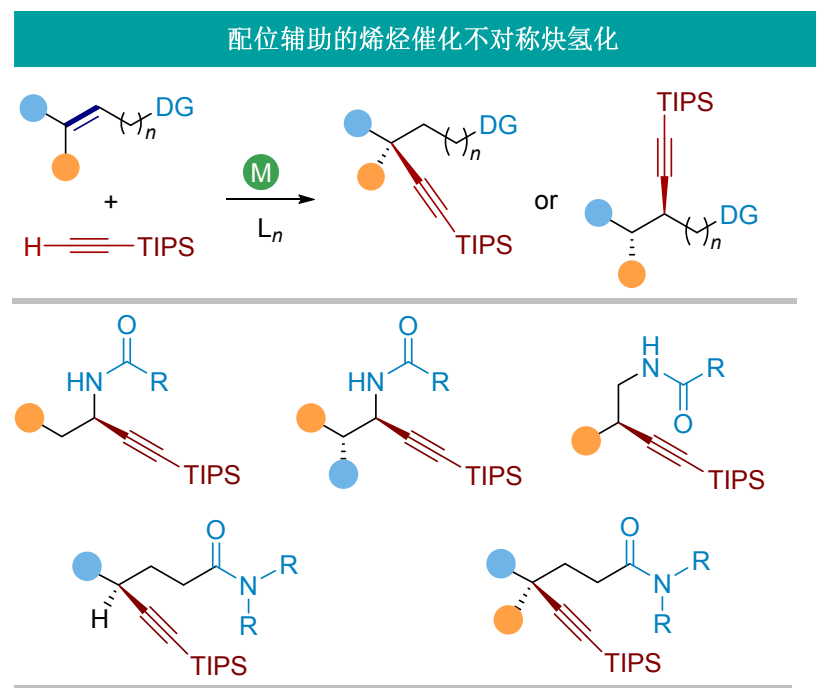

图 3 配位辅助的烯烃炔氢化小结

Figure 3 Overview of substrate-directed asymmetric hydroalkynylations

\section{2 烯烃的催化不对称炔氢化}

\section{1 铑催化烯基酰胺 $\alpha$ 位不对称炔氢化}

我们的研究起始于烯基酰胺类化合物的不对称炔 氢化. 烯基酰胺是一类常见的化合物 ${ }^{[16]}$, 可以通过烯基 卤代物的偶联反应及炔烃的氢胺化反应制备 ${ }^{[17]}$. 烯基 酰胺两个反应位点的炔基化分别生成炔丙基胺及高炔 丙基胺化合物，两者都是有用的合成单元. 此外，烯基 酰胺中的酰胺基团可以与过渡金属进行配位，起到稳定 过渡金属中间体或降低反应过渡态能量的作用(图 4)。 
<smiles>[R]CC(C#C[In]S)NC([R])=O</smiles><smiles>[R]C/C=C\NC([R])=O</smiles>

图 4 烯基酰胺选择性炔氢化设计

Figure 4 Design on selective hydroalkynylation of enamide

此类反应的难点在于烯基酰胺是富电子烯烃, 而端 炔形式上是亲核试剂, 二者之间的反应在电性上并不有 利. 在我们的反应设计中, 通过端炔与阳离子低价金属 的氧化加成生成一个高价的亲电性金属物种, 从而有利 于后续与富电子烯基酰胺的反应. 该炔基金属氢物种与 烯基酰胺有两种可能的反应途径. 一是先发生烯烃对金 属氢的迁移插入, 再通过后续的碳碳键还原消除得到相 应的炔丙基胺化合物(Chalk-Harrod 机理) ${ }^{[18]}$. 另一个途 径是先发生烯烃对金属碳键的迁移插入, 再通过后续的 碳氢键还原消除得到相应的高炔丙基胺化合物(修正的 Chalk-Harrod 机理 $)^{[19]}$. 因此, 迁移插入过程决定了烯烃 炔氢化反应的选择性.

研究发现, 当我们使用阳离子铑作催化剂、富电子 双膦配体 ${ }^{i} \mathrm{Pr}$-MeOBIPHEP 作配体时, 以单一的区域选 择性和很高的对映选择性得到手性的炔丙基酰胺产 物 ${ }^{[20]}$. 该方法为手性炔丙基胺类化合物的制备提供了 一种新的思路 ${ }^{[21]}$. 该反应对于一系列的硅基、大位阻的 烷基和芳基取代的端炔能够顺利发生; 但是, 小位阻的 端炔反应性较低. 一系列不同官能团取代的烯基酰胺均 有很好的反应性和较高的对映选择性(图 5).

我们研究了炔氢化的非对映选择性, 结果表明该炔 基化过程具有立体专一性. 炔烃与烯基酰胺的反应是同 面加成过程，因而排除了经由亚胺中间体的机理.

进一步的机理研究表明, 催化剂以烯基酰胺配位的 阳离子铑为主要存在形式. 动力学测试表明该反应对催 化剂是一级, 对炔烃也是一级, 而对烯胺是零级反应. 氞代炔烃的动力学同位素实验结果表明, KIE 为 $1.5 \pm$ 0.2 . 以上这些结果均支持烯烃的迁移插入而非炔烃的
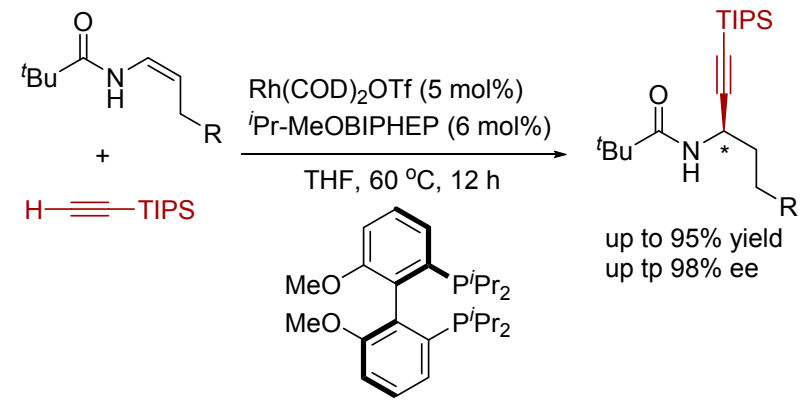
up tp $98 \%$ ee

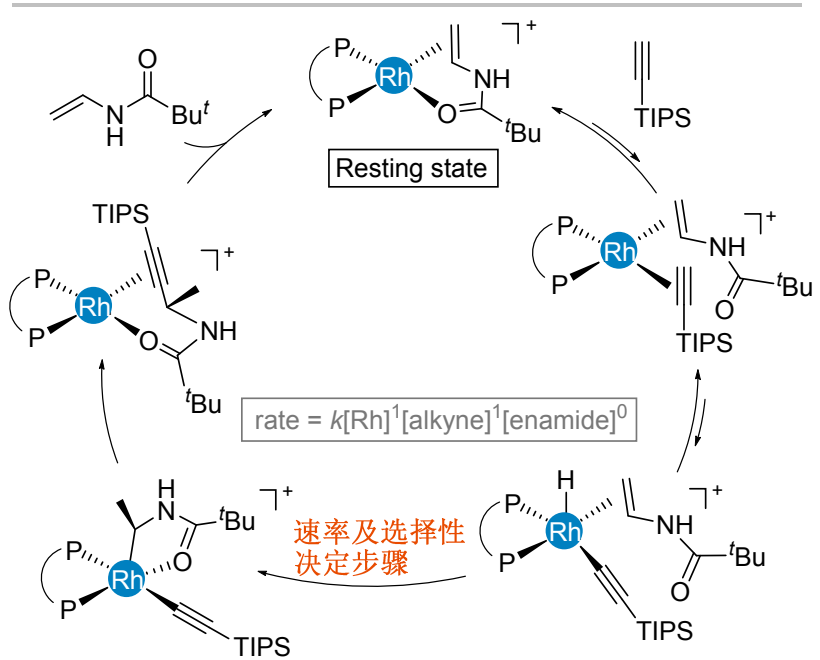

图 5 烯基酰胺不对称 $\alpha$ 炔氢化及机理

Figure 5 Asymmetric $\alpha$-hydroalkynylation of enamide and its mechanism

$\mathrm{C}-\mathrm{H}$ 键断裂是反应的决速步.

计算化学的结果表明, 烯烃的迁移插入步骤具有最 高的活化能，这一不可逆的过程是反应决速步及对映选 择性决定步骤. 生成主要对映异构体的过渡态中, 配体 与底物间的排斥更小, 并且配体与底物形成的 $\mathrm{C}$ $\mathrm{H} \cdots \mathrm{O}$ 相互作用较强. 因此, 配体结构在控制烯烃迁移 插入步骤的能量起到了关键作用.

\section{2 铱催化烯基酰胺 $\beta$ 位不对称炔氢化}

烯基烯胺类化合物炔氢化的另一个可能的产物是 经由 $\beta$ 位炔基化得到高炔丙基胺. 在前述反应设计的分 析中, 如果能够使烯烃迁移插入的方式发生改变, 则可 以实现区域选择性的逆转. 我们设想, 将金属中心从铑 改变为铱时, 由于金属铱中心较难发生碳碳键还原消 除 ${ }^{[22]}$, 从而使得烯烃迁移插入至金属氢键的途径不利, 反应有可能按照烯烃迁移插入至金属碳键的途径进行.

实验发现，当使用阳离子铱作催化剂、富电子双膦 配体 BPE-Ph 作配体时，以单一的区域选择性和很高的 对映选择性得到手性的高炔丙基酰胺产物，反应中并没 有观察到任何的炔丙基胺产物 ${ }^{[23]}$. 该方法提供了一种 合成 $\beta$ 位取代的高炔丙基胺的新策略 ${ }^{[24]}$. 反应对官能团 有较好的兼容性. 产物中的炔基可以进行多种类型的转 
换，从而得到一系列手性的胺类化合物(图 6).

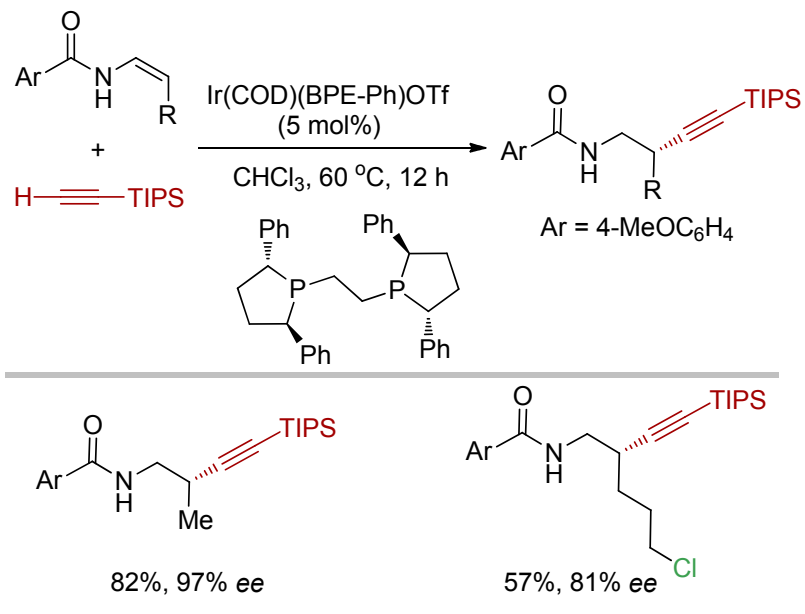<smiles>CC/C=C/CCC(C#C[In])CNC(=O)Br</smiles><smiles>O=C([Al])NC[C](C#C[In])CCC(=O)c1ccccc1</smiles>

$68 \%, 87 \%$ ee<smiles>O=C(Br)NC[C@H](C#C[In])CCC[TeH-]</smiles>

$64 \%, 93 \%$ ee

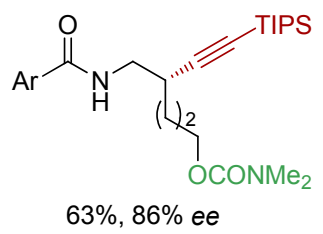

图 6 烯基酰胺不对称 $\beta$ 炔氢化

Figure 6 Asymmetric $\beta$-hydroalkynylation of enamide

林正扬课题组 ${ }^{[25]}$ 对这一体系的机理进行了计算. 计算结果表明, 烯烃对铱氢键的插入可以顺利发生, 但 后续铱中心碳碳键的还原消除过渡态能量很高, 不能顺 利进行. 相比而言, 烯烃对于铱碳键的迁移插入虽然比 烯烃对于铱氢键的迁移插入能量稍高, 但后续铱中心碳 氢键还原消除的能量比相应碳碳键还原消除的能量低 很多. 因此, 最终反应朝着烯烃迁移插入到铱碳键的方 向进行，从而得到 $\beta$ 位炔基化的产物.

\section{3 铱催化 $\beta, \beta$-双取代烯基酰胺不对称炔氢化构建连 续手性中心}

在单取代烯基酰胺不对称炔氢化构建单个手性中 心的基础上, 我们进一步发展新的催化体系以实现二取 代烯基酰胺的不对称炔氢化构建连续手性中心. 二取代 烯基酰胺较单取代烯基酰胺位阻更大，因此其反应性更 低. 此外反应产生了两个手性中心，因而涉及到非对映 选择性的控制. 我们发展的低价金属催化的不对称炔氢 化反应具有同面加成的立体化学特征, 其固有的立体专 一性即能保证非对映选择性得到很好的控制.

从 $\beta, \beta$-二取代的烯基酰胺出发, 我们尝试了阳离子
铑络合物与一系列的配体以实现其 $\alpha$ 位炔基化，但均没 有得到目标产物，这也反映了 $\beta, \beta$-二取代的烯基酰胺比 单取代烯基酰胺更低的反应性. 我们转而尝试阳离子铱 催化剂. 研究发现, 以阳离子铱作催化剂、Carreira 类型 亚磷酰胺 ${ }^{[26]}$ 为配体，实现了 $\beta, \beta$-双取代烯基酰胺的不对 称炔氢化，以单一的 $\alpha$ 位选择性、>20:1 的非对映选 择性及高达 99\% ee 值的对映选择性得到炔丙基胺类化 合物，高效构建两个手性中心 ${ }^{[27]}$. 亚磷酰胺配体对反应 的进行至关重要，许多其他类型的富电子膦配体均只有 很低的反应性. 基于该催化体系的炔氢化选择性发生在 $\alpha$ 位，说明除了催化中心金属的特性以外，配体也在很 大程度上参与了反应选择性的调控(图 7).

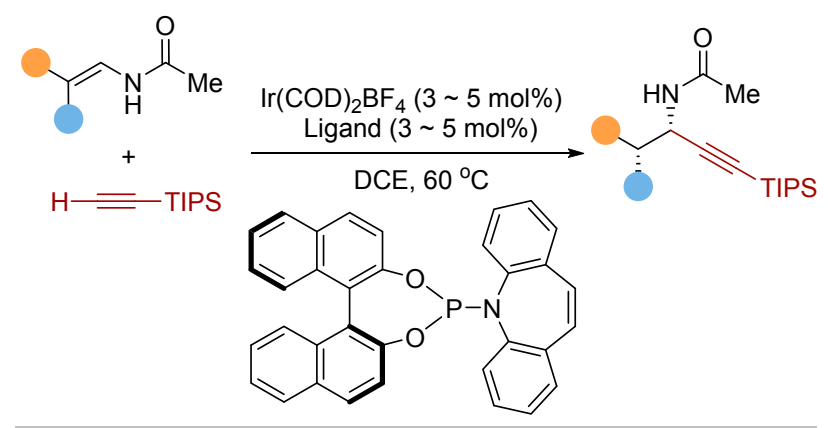

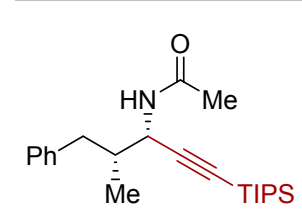

$97 \%,>99 \%$ ee, $d r>20: 1$

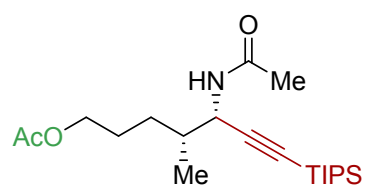

$96 \%,>99 \%$ ee, $d r>20: 1$

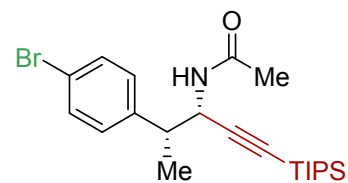

$65 \%,>99 \%$ ee, $d r>20: 1$

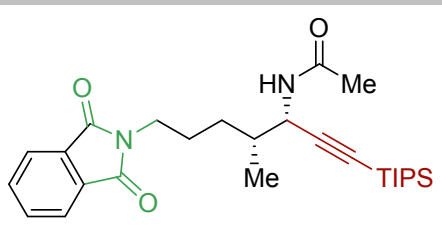

$99 \%, 99 \%$ ee, $d r>20: 1$

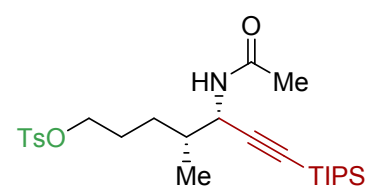

$99 \%,>99 \%$ ee, $d r>20: 1$

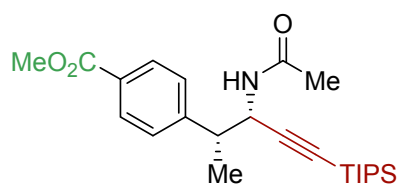

$64 \%,>99 \%$ ee, $d r>20: 1$
图 7 烯基酰胺不对称 $\alpha$ 炔氢化构建连续手性中心

Figure 7 Asymmetric $\alpha$-hydroalkynylation of enamide to create vicinal stereocenters

由于该催化炔氢化过程是立体专一性的，因此通过 改变烯基酰胺的构型就能实现非对映选择性的控制. 从 $E$ 型烯基酰胺出发, 在标准条件下得到 $s y n$ 型炔氢化产 物. 改变烯基酰胺的构型，从 $Z$ 型烯基酰胺出发，同样 条件下即能得到单一的 anti-产物. 使用另一个构型的配 体，从 $E$ 和 $Z$-烯基酰胺进行炔氢化则得到以上两个产物 
的对映体(图 8). 因此, 利用烯烃构型及配体构型的组 合，即可以高非对映选择性、高对映选择性实现四个立 体异构体的立体多样性合成 ${ }^{[28]}$.

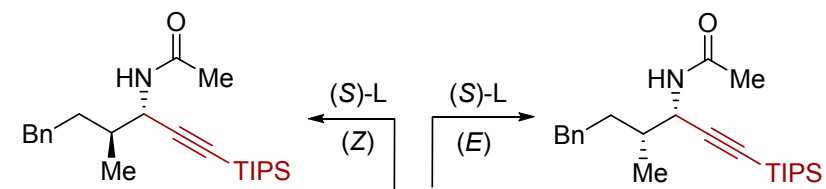

$(S, S) 83 \%,>99 \%$ ee
$d r=20: 1$<smiles>CC(=O)NC=C(C)CBr</smiles>

$(S, R) 99 \%,>99 \%$ ee $d r>20: 1$<smiles>CC(=O)N[C@@H](C#C[In])[C@H](C)CBr</smiles>

$(R, R) 82 \%,>99 \%$ ee $d r>20: 1$

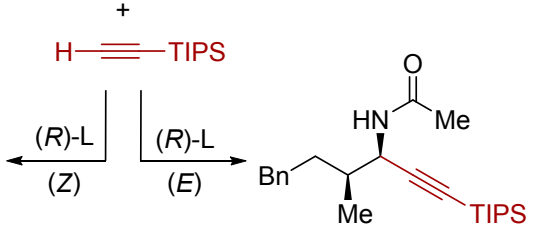

$(R, S) 99 \%,>99 \%$ ee $d r>20: 1$
图 8 不对称炔氢化用于立体多样性合成

Figure 8 Asymmetric hydroalkynylation for stereodivergent synthesis

通过测试不同催化剂与配体比例的催化活性及非 线性效应的实验结果, 表明催化体系中金属与配体以 1:1 形式配位. 在此基础上, 我们进行了计算化学的研 究以分辨催化过程是通过 Chalk-Harrod机理还是修正的 Chalk-Harrod 机理进行 ${ }^{[18,19]}$. 两组关键的数据支持该反 应是通过 Chalk-Harrod 机理进行的. 首先, 两个催化途 径的能量相比, Chalk-Harrod 机理最高过渡态的能量 $(111 \mathrm{~kJ} / \mathrm{mol})$ 比修正的 Chalk-Harrod 机理最高过渡态的 能量 $(141 \mathrm{~kJ} / \mathrm{mol})$ 低, 而且与实验条件(温度为 $60{ }^{\circ} \mathrm{C}$ )更 加吻合. 其次, 通过 Chalk-Harrod 机理计算得到的主要 对映体与实验结果相一致, 而通过修正的 Chalk-Harrod 机理预测的主要对映体则与实验结果相反. 在 Chalk-Harrod 机理中, 决速步铱中心的碳碳键还原消除 步骤能量较低, 配体对此起到了关键作用. 亚磷酰胺类 配体比双膦配体更贫电子，因此金属中心的电子云密度 降低，从而有利于铱中心发生还原消除(图 9).

\section{4 铱催化 $\alpha, \beta$-及 $\beta, \gamma$-不饱和酰胺 $\gamma$ 位不对称炔氢化}

以上铱催化的烯基酰胺不对称炔氢化机理研究的 结果可用于指导新型烯烃不对称炔氢化反应的设计. 例 如, 从非活化烯烃 $\beta, \gamma$-不饱和酰胺出发, 使用铱催化剂 及较富电子双磷配体, 反应经由修正的 Chalk-Harrod 机 理即可得到羰基 $\gamma$ 位炔基化的产物. 若该反应成功实现, 将为羰基远端手性中心的构建提供新的方法 ${ }^{[29]}$, 并为 配位辅助的非活化烯烃的区域及立体选择性氢官能团 化提供新的思路.
Chalk-Harrod mechanism

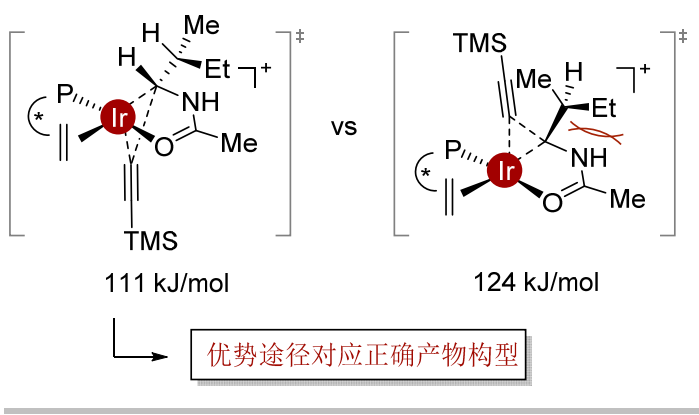

Modified Chalk-Harrod mechanism

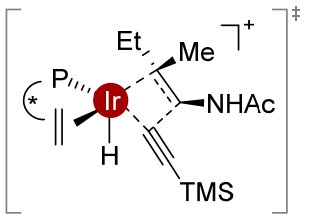

$141 \mathrm{~kJ} / \mathrm{mol}$

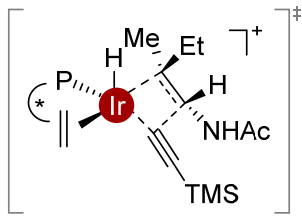

$147 \mathrm{~kJ} / \mathrm{mol}$

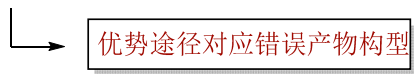

图 9 铱催化不对称 $\alpha$ 炔氢化机理

Figure 9 Mechanism of Ir-catalyzed asymmetric $\alpha$-hydroalkynylation of enamide

实验结果表明，当使用阳离子铱作催化剂、富电子 双膦配体 DM-Segphos 作配体时，以单一的区域选择性 和较高的对映选择性得到手性的 $\gamma$-炔基酰胺 ${ }^{[30]}$. 进一步 监测反应体系发现, 部分 $\beta, \gamma$-不饱和酰胺异构化为 $\alpha, \beta$ 不饱和酰胺, 但最终二者都转化为相应的 $\gamma$-炔基酰胺产 物(图 10). 相应的 $\beta, \gamma$-不饱和酯及 $\alpha, \beta$-不饱和酯类化合 物不能发生相应的反应，说明酰胺的配位作用对反应的 进行至关重要.

因此, 我们直接从 $\alpha, \beta$-不饱和酰胺出发, 在同样的 条件下即可实现其 $\gamma$ 位不对称炔基化反应，形式上实现 了氧化还原中性的 $\alpha, \beta$-不饱和酰胺 $\gamma$ 位不对称碳氢键官 能团化 ${ }^{[31]}$. 特别需要指出的是, 在该催化体系中, 并没 有发生共轭加成的炔基化产物，我们认为是由于中间体 中铱中心难以直接发生碳碳键还原消除, 转而进行 $\beta$-氢 消除得到烯烃异构化的产物再发生后续的碳碳键迁移 插入及碳氢键还原消除(Chalk-Harrod 机理), 从而选择 性的得到酰胺远端炔基化的产物.

\section{5 铱催化 $\gamma, \gamma$-二取代 $\beta, \gamma$-不饱和酰胺炔氢化构建全 碳季碳手性中心}

全碳季碳手性中心的高效构建是不对称合成中的 挑战之一 ${ }^{[32]}$. 在实现了酰胺辅助的烯烃不对称炔氢化 构建三级碳中心的基础上, 我们发展新的催化体系, 进 一步实现了全碳季碳手性中心的构建. 

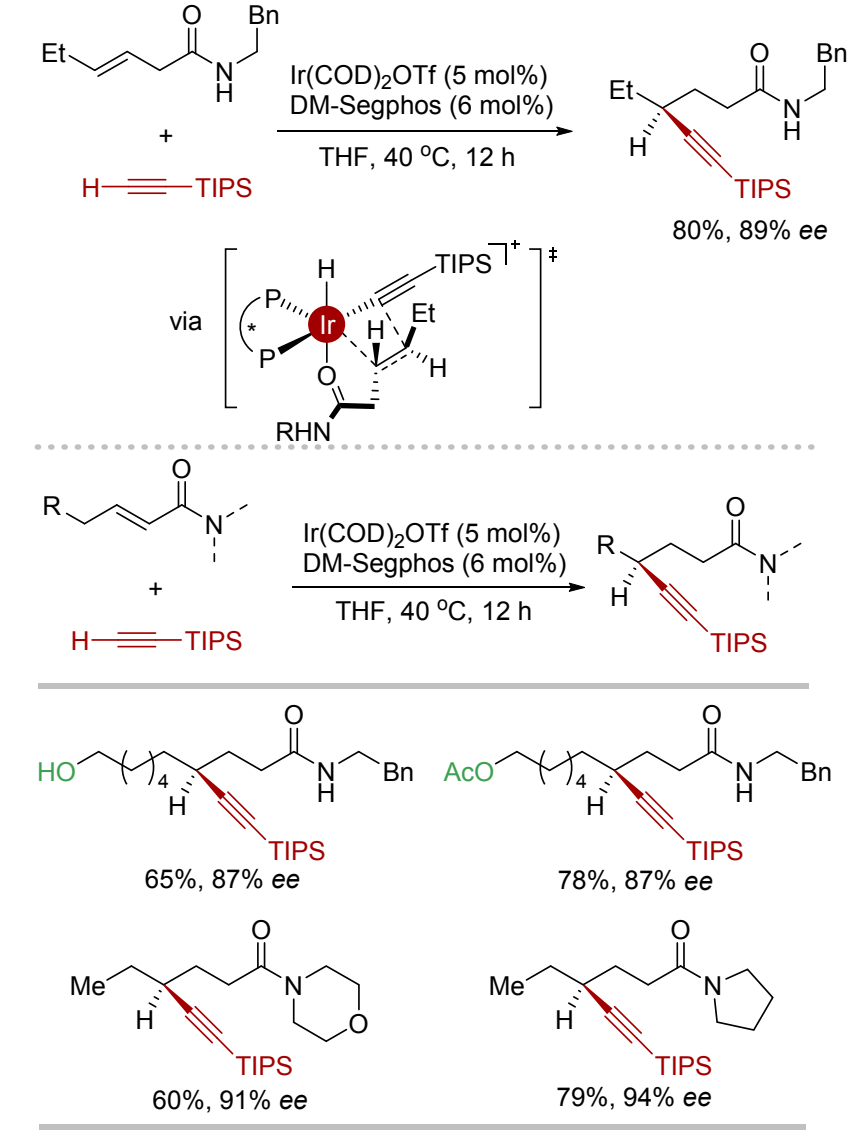

$80 \%, 89 \%$ ee

图 10 铱催化不饱和酰胺不对称 $\gamma$ 炔氢化

Figure 10 Ir-catalyzed asymmetric $\gamma$-hydroalkynylation of unsaturated amides

从三取代非活化烯烃出发通过不对称炔氢化构建 季碳手性中心面临几个方面的挑战. 首先, 三取代烯烃 较二取代烯烃位阻更大, 形成季碳手性中心更加困难. 其次, 炔氢化的区域选择性需要加以控制, 否则反应有 可能发生在位阻更小的一端. 另外, 还需抑制烯烃异构 化过程, 因为原料烯烃构型的变化将直接导致对映选择 性的下降.

实验结果表明(图 11), 以三取代烯烃 $\beta, \gamma$-不饱和酰 胺为原料, 当使用阳离子铱作催化剂、双膦配体 CTH-P-Phos 作配体时, 以单一的 $\gamma$ 位区域选择性和较高 的对映选择性得到手性的炔基化产物, 从非官能团化的 原料出发高效构建全碳季碳手性中心 ${ }^{[33]}$. 在这个催化 体系中, 三级酰胺比二级酰胺表现出更好的反应性, 具 体原因还有待进一步研究.

虽然目前我们发展的几类炔氢化反应中炔烃主要 局限于硅基端炔, 但硅基非常容易脱除, 所得到的端炔 可以进行多种转化. 例如, 含全碳季碳手性中心的产物 脱除硅基后再进行多种类型的后续转化, 从而合成一系 列含季碳手性中心的分子. 炔基进行半氢化、氢化、 Sonogashira 偶联、Click 反应、吲哚环化、氧化断裂等,
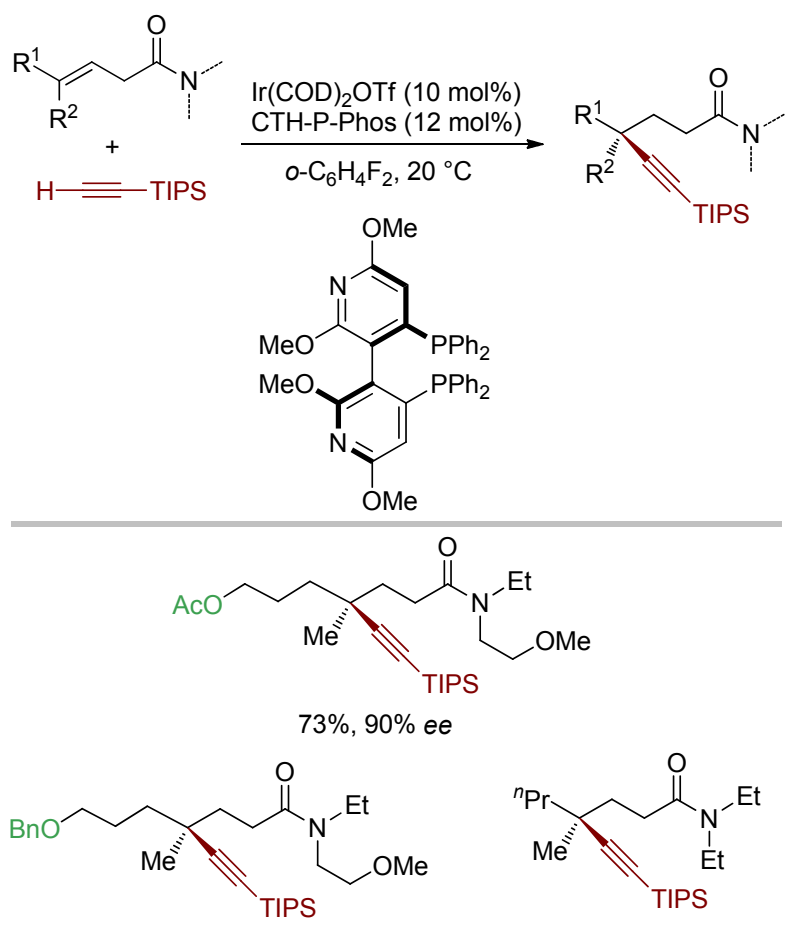

$72 \%, 93 \%$ ee $65 \%, 90 \%$ ee<smiles>CCN(CCc1ccco1)C(=O)CC[C@](C)(C#C[In])C(C)C</smiles>

$64 \%, 89 \%$ ee

图 11 铱催化三取代烯烃炔氢化构建季碳中心 Figure 11 Ir-catalyzed asymmetric hydroalkynylation of tri-substituted alkene to construct quaternary carbon centers

分别得到含烯基、烷基、炔基、杂环及羧基的季碳手性 中心，实现了手性分子的多样性合成(图 12).

催化体系中催化剂主要存在形式及动力学同位素 效应实验的研究表明, 烯烃迁移插入至铱碳键是反应的 决速步. 进一步的计算结果也支持了这一结论; 但计算 预测反应的对映选择性应在 $99 \%$ ee 以上，这与实验结 果有一定差别. 后续的研究发现, 原料中烯烃的异构化 是导致对映选择性下降的原因. 监测反应体系发现, 随 着反应的进行, 原料的 $E / Z$ 比例逐渐下降, 产物的 $e e$ 值 也逐步下降，二者之间存在一定的相关性，说明烯烃迁 移插入步骤的面选择性及烯烃异构化过程共同控制了 反应的对映选择性(图 13).

配位基团酰胺的存在对反应的进行及立体选择性 的控制至关重要，相应含酯基的烯烃底物则没有反应 性. 通过计算相应酰胺与金属中心不进行配位的烯烃迁 移插入过渡态发现，此类迁移插入过渡态的能量比相应 酰胺与金属中心进行配位的过渡态能量高出很多，说明 酰胺通过与金属中心的配位大大降低了烯烃迁移插入 这一决速步的过渡态能量. 
<smiles>[R]CC(C)(C)CCC(=O)N([R])CC</smiles>

$79 \%, 92 \%$ ee<smiles>[Y19]C[C@](C)(CCBr)CCC(=O)N([R])CC</smiles>

$91 \%, 92 \%$ ee<smiles>[R]N(CC)C(=O)CCC(C)(CCBr)c1cn[nH]n1</smiles>

Ts

$86 \%, 92 \%$ ee

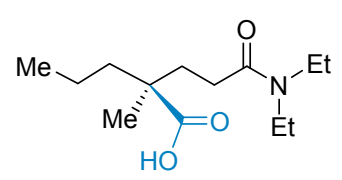

$57 \%$
图 12 炔基取代季碳中心的转化

Figure 12 Transformations of alkyne substituted quaternary carbon centers

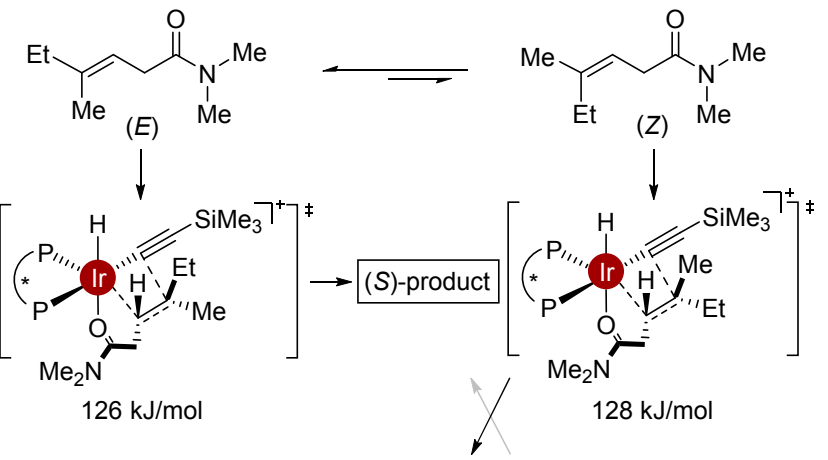

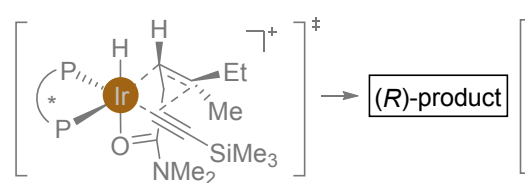

$148 \mathrm{~kJ} / \mathrm{mol}$

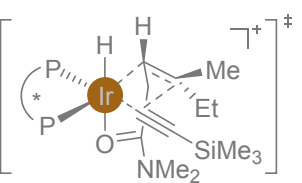

$149 \mathrm{~kJ} / \mathrm{mol}$
图 13 对映选择性决定因素

Figure 13 Controlling factors of enantioselectivity

\section{3 结论}

配位辅助是烯烃不对称催化转化中实现选择性控 制的一类十分有效的策略. 通过底物中配位基团与金属 中心的配位作用，提升烯烃的反应性，并调控烯烃的反 应位点及反应面, 实现烯烃转化中区域选择性及立体选 择性的高效控制. 通过使用配位辅助策略，我们发展了 烯烃催化转化的新型反应, 实现了温和条件下烯烃高区 域选择性、高立体选择性的催化转化, 并通过实验与计
算相结合, 研究了烯烃转化中区域选择性及立体选择性 的控制因素，为烯烃的高效利用提供科学依据.

虽然配位辅助的烯烃不对称转化领域取得了一些 进展，但目前该方向仍然有许多问题有待进一步深入研 究. 例如, 基于配位辅助的不对称碳碳键形成反应类型 还十分有限, 氢官能团化(hydrofunctionalization)相比于 质子官能团化 (protofunctionalization) 反应 ${ }^{[34]}$ 更加稀少; 配位基团的实用性有待进一步提升，如羟基、胺基、羰 基等官能团作为配位基团的烯烃转化需要进一步开发; 另外, 反应机理的研究仍需进一步深入，并基于机理的 研究进而指导新型、高效催化体系的发现. 相信经过化 学家的持续努力, 配位辅助的烯烃不对称转化领域将取 得更大的进展，并催生烯烃不对称催化新方法、新策略 的发现及应用.

\section{作者简介}

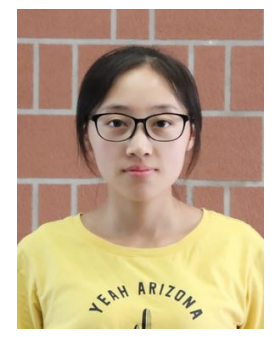

张雯雯, 2016 年在河南师范大学获得学士学位, 2019 年在中国人民大学获得硕士学位, 目前在清华大 学基础分子科学中心李必杰课题组攻读博士学位.

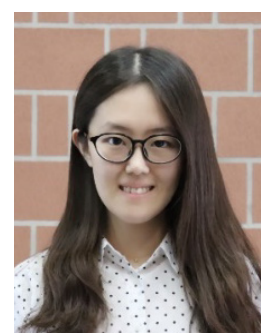

王紫璇, 2016 年在北京化工大学获得学士学位, 目 前在清华大学基础分子科学中心李必杰课题组攻读博 士学位.

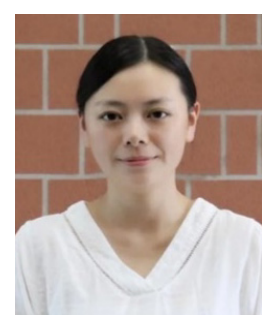

白小燕, 2012 年在西昌学院获得学士学位, 2015 年 在四川师范大学获得硕士学位, 目前在清华大学基础分 子科学中心李必杰课题组攻读博士学位. 


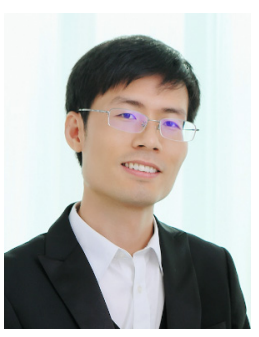

李必杰, 清华大学化学系副教授, 博士生导师. 分 别于 2007 年及 2012 年在北京大学化学学院获得本科及 博士学位, 之后在加州大学伯克利分校开展博士后研 究, 2015 年加入清华大学化学系基础分子科学中心开展 独立工作, 主要研究方向为不对称催化及烯烃转化. 入 选 2015 年中组部青年千人计划, 获得了 2017 年 Thieme Chemistry Journal Award 及 2019 年中国化学会青年化学 奖.

\section{References}

[1] Wang, J. Stereoselective Alkene Synthesis; Springer-Verlag, Berlin/Heidelberg, 2012.

[2] (a) Coombs, J. R.; Morken, J. P. Angew. Chem. Int. Ed. 2016, 55, 2636.

(b) Chen, J. H.; Lu, Z. Org. Chem. Front. 2018, 5, 260

(c) Dhungana, R. K.; Kc, S.; Basnet, P.; Giri, R. Chem. Rec. 2018, $18,1314$.

(d) Zhang, J.-S.; Liu, L.; Chen, T.; Han, L.-B. Chem. Asian J. 2018, 13, 2277.

(e) Ping, Y.; Li, Y.; Zhu, J.; Kong, W. Angew. Chem., Int. Ed. 2019, 58,1562 .

(f) Li, S.; Li, Z.; You, C.; Lü, H.; Zhang, X. Chin. J. Org. Chem. 2019, 39, 1568.

(g) Cheng, L.; Xie, J. Chin. J. Org. Chem. 2020, 40, 247.

(h) Zhou, Q.; Feng, X,; Yang, J.; Du, H. Chin. J. Org. Chem. 2019, $39,2188$.

[3] Wang, Z.-X.; Bai, X.-Y.; Li, B.-J. Chin. J. Chem. 2019, 37, 1174.

[4] (a) Knöpfel, T. F.; Zarotti, P.; Ichikawa, T.; Carreira, E. M. J. Am. Chem. Soc. 2005, 127, 9682 .

(b) Nishimura, T.; Guo, X.-X.; Uchiyama, N.; Katoh, T.; Hayashi, T. J. Am. Chem. Soc. 2008, 130, 1576

(c) Yazaki, R.; Kumagai, N.; Shibasaki, M. J. Am. Chem. Soc. 2010, 132,10275

(d) Shirakura, M.; Suginome, M. Angew. Chem. Int. Ed. 2010, 49, 3827.

(e) Fan, B.-M.; Yang, Q.-J.; Hu, J.; Fan, C.-L.; Li, S.-F.; Yu, L.; Huang, C.; Tsang, W. W.; Kwong, F. Y. Angew. Chem., Int. Ed. 2012, 51,7821 .

(f) Sawano, T.; Ou, K.; Nishimura, T.; Hayashi, T. Chem. Commun. 2012, 48, 6106.

(g) Blay, G.; Pedro, J. R.; Sanz-Marco, A. Synthesis 2018, 50, 3281. (h) Zhi, Y.; Huang, J.; Liu, N.; Lu, T.; Dou, X. Org. Lett. 2017, 19, 2378.

[5] (a) Fu, L.; Zhou, S.; Wan, X.; Chen, P.; Liu, G. J. Am. Chem. Soc. 2018, 140, 10965.

(b) Yeqiang, H.; Ding, Y.; Zhou, T.; Yan, S.-Y.; Song, H.; Shi, B.-F. J. Am. Chem. Soc. 2019, 141, 4558.

(c) Dong, X.-Y.; Zhang, Y.-F.; Ma, C.-L.; Gu, Q.-S.; Wang, F.-L.; Li, Z.-L.; Jiang, S.-P.; Liu, X.-Y. Nat. Chem. 2019, 11, 1158.

(d) Chen, Q.; Tang, Y.; Huang, T.; Liu, X.; Lin, L.; Feng, X. Angew. Chem., Int. Ed. 2016, 55, 5286.

[6] (a) Trost, B. M., Li, C.-J. Modern Alkyne Chemistry: Catalytic and Atom-Economic Transformations, Wiley, New York, 2014.

(b) Li, Y.; Liu, X.; Jiang, H.; Liu, B.; Chen, Z.; Zhou, P. Angew.
Chem., Int. Ed. 2011, 50, 6341

(c) Sivaguru, P.; Wang, Z.; Zanoni, G.; Bi, X. Chem. Soc. Rev. 2019, 48, 2615.

(d) Hong, F.-L.; Wang, Z.-S.; Wei, D.-D.; Zhai, T.-Y.; Deng, G.-C.; Lu, X.; Liu, R.-S.; Ye, L.-W. J. Am. Chem. Soc. 2019, 141, 16961.

(e) Zhu, D.; Chen, L.; Zhang, H.; Ma, Z.; Jiang, H.; Zhu, S. Angew. Chem., Int. Ed. 2018, 57, 12405.

(f) Ren, R.; Wu, Z.; Xu, Y.; Zhu, C. Angew. Chem., Int. Ed. 2016, $55,2866$.

(g) Shen, T.; Wang, T.; Qin, C.; Jiao, N. Angew. Chem., Int. Ed. 2013, 52, 6677.

(h) Xu, G.; Zhao, H.; Fu, B.; Cang, A.; Zhang, G.; Zhang, Q.; Xiong, T.; Zhang, Q. Angew. Chem., Int. Ed. 2017, 56, 13130.

(i) Wang, Q.; Yu, X.; Jin, J.; Wu, Y.; Liang, Y. Chin. J. Chem. 2018, $36,223$.

(j) Jiang, Z.; Lu, P.; Wang, Y. Org. Lett. 2012, 14, 6266.

(k) Zhu, C.; Chu, H.; Li, G.; Ma, S.; Zhang, J. J. Am. Chem. Soc. 2019, 141, 19246.

[7] Diederich, F.; Stang, P. J.; Tykwinski, R. R. Acetylene Chemistry, Wiley-VCH, Weinheim, 2005.

[8] (a) Simmons, H. E.; Smith, R. D. J. Am. Chem. Soc. 1959, 81, 4256.

(b) Winstein, S.; Sonnenberg, J.; De Vries, L. J. Am. Chem. Soc. 1959, 81, 6523.

(c) Winstein, S.; Sonnenberg, J. J. Am. Chem. Soc. 1961, 83, 3235.

[9] Henbest, H. B.; Wilson, R. A. L. J. Chem. Soc. 1957, 1958.

[10] (a) Thompson, H. W.; McPherson, E. J. Am. Chem. Soc. 1974, 96, 6232.

(b) Crabtree, R. H.; Davis, M. W. J. Org. Chem. 1986, 51, 2655.

[11] (a) Tang, W.; Zhang, X. Chem. Rev. 2003, 103, 3029.

(b) Zhu, S.-F.; Zhou, Q.-L. Acc. Chem. Res. 2017, 50, 988.

[12] (a) Evans, D. A.; Fu, G. C. J. Am. Chem. Soc. 1991, 113, 4042

(b) Rubina, M.; Rubin, M.; Gevorgyan, V. J. Am. Chem. Soc. 2003, 125,7198 .

(c) Bochat, A. J.; Shoba, V. M.; Takacs, J. M. Angew. Chem., Int. Ed. 2019, 58, 9434.

[13] Kawasaki, Y.; Ishikawa, Y.; Igawa, K.; Tomooka, K. J. Am. Chem. Soc. 2011, 133, 20712.

[14] (a) Willis, M. C. Chem. Rev. 2010, 110, 725.

(b) Murphy, S. K.; Bruch, A.; Dong, V. M. Angew. Chem., Int. Ed. 2014, 53, 2455.

[15] (a) Albrecht, M. Chem. Rev. 2010, 110, 576.

(b) Lyons, T. W.; Sanford, M. S. Chem. Rev. 2010, 110, 1147.

(c) He, J.; Wasa, M.; Chan, K. S. L.; Shao, Q.; Yu, J.-Q. Chem. Rev. 2017, 117, 8754.

(d) Li, B.-J.; Shi, Z.-J. Chem. Soc. Rev. 2012, 41, 5588.

[16] Wang, M.-X. Chem. Commun. 2015, 51, 6039.

[17] (a) Jiang, L.; Job, G. E.; Klapars, A.; Buchwald, S. L. Org. Lett. 2003, $5,3667$.

(b) Gooßen, L. J.; Rauhaus, J. E.; Deng, G. Angew. Chem., Int. Ed. $\mathbf{2 0 0 5}, 44,4042$.

[18] Sakaki, S.; Mizoe, N.; Sugimoto, M. Organometallics 1998, 17, 2510.

[19] Sakaki, S.; Sumimoto, M.; Fukuhara, M.; Sugimoto, M.; Fujimoto, H.; Matsuzaki, S. Organometallics 2002, 21, 3788.

[20] Bai, X. Y.; Zhang, W. W.; Li, Q.; Li, B.-J. J. Am. Chem. Soc. 2018, 140, 506.

[21] Peshkov, V. A.; Pereshivko, O. P.; Van der Eycken, E. V. Chem. Soc. Rev. 2012, 41, 3790.

[22] Hartwig, J. F. Organotransition Metal Chemistry: From Bonding to Catalysis, University Science Books, Sausalito, CA, 2010.

[23] Bai, X.-Y.; Wang, Z.-X.; Li, B.-J. Angew. Chem., Int. Ed. 2016, 55, 9007.

[24] Ding, C. H.; Hou, X. L. Chem. Rev. 2011, 111, 1914.

[25] Yu, Z.; Meng, L.; Lin, Z. Organometallics 2019, 38, 2998.

[26] Rössler, S. L.; Petrone, D. A.; Carreira, E. M. Acc. Chem. Res. 2019, 52, 2657.

[27] Zhang, W.-W.; Zhang, S.-L.; Li, B.-J. Angew. Chem., Int. Ed. 2020, 
$59,6874$.

[28] (a) Krautwald, S.; Carreira, E. M. J. Am. Chem. Soc. 2017, 139, 5627.

(b) Huo, X.; He, R.; Zhang, X.; Zhang, W. J. Am. Chem. Soc. 2016, 138, 11093.

(c) Wei, L.; Zhu, Q.; Xu, S.-M.; Chang, X.; Wang, C.-J. J. Am. Chem. Soc. 2018, 140, 1508.

(d) Zhang, Q.; Yu, H.; Shen, L.; Tang, T.; Dong, D.; Chai, W.; Zi, W. J. Am. Chem. Soc. 2019, 141, 14554.

[29] (a) Mei, T.-S.; Patel, H. H.; Sigman, M. S. Nature 2014, 508, 340. (b) Liu, W.-B.; Okamoto, N.; Alexy, E. J.; Hong, A. Y.; Tran, K.; Stoltz, B. M. J. Am. Chem. Soc. 2016, 138, 5234.

(c) Guo, C.; Fleige, M.; Janssen-Muller, D.; Daniliuc, C. G.; Glorius, F. J. Am. Chem. Soc. 2016, 138, 7840.

[30] Wang, Z.-X.; Bai, X.-Y.; Yao, H.-C.; Li, B.-J. J. Am. Chem. Soc. 2016, $138,14872$.

[31] Li, Y.; Wu, D.; Cheng, H.-G.; Yin, G. Angew. Chem., Int. Ed. 2020, $59,7990$.

[32] (a) Quasdorf, K. W.; Overman, L. E. Nature 2014, 516, 181. (b) Feng, J.; Holmes, M.; Krische, M. J. Chem. Rev. 2017, 117, 12564 .

(c) Zeng, X.-P.; Cao, Z.-Y.; Wang, Y.-H.; Zhou, F.; Zhou, J. Chem. Rev. 2016, 116, 7330.

[33] Wang, Z.-X.; Li, B.-J. J. Am. Chem. Soc. 2019, 141, 9312.

[34] (a) Wang, H.; Bai, Z.; Jiao, T.; Deng, Z.; Tong, H.; He, G.; Peng, Q.; Chen, G. J. Am. Chem. Soc. 2018, 140, 3542.

(b) Liu, Z.; Li, X.; Zeng, T.; Engle, K. M. ACS Catal. 2019, 3260.

(c) Shen, H.-C.; Zhang, L.; Chen, S.-S.; Feng, J.; Zhang, B.-W.; Zhang, Y.; Zhang, X.; Wu, Y.-D.; Gong, L.-Z. ACS Catal. 2019, 9, 791.

(d) Nimmagadda, S. K.; Liu, M.; Karunananda, M. K.; Gao, D. W.; Apolinar, O.; Chen, J. S.; Liu, P.; Engle, K. M. Angew. Chem., Int. Ed. 2019, 58, 3923.

(e) Vanable, E. P.; Kennemur, J. L.; Joyce, L. A.; Ruck, R. T.; Schultz, D. M.; Hull, K. L. J. Am. Chem. Soc. 2019, 141, 739.

(f) Tang, C.; Zhang, R.; Zhu, B.; Fu, J.; Deng, Y.; Tian, L.; Guan, W.; Bi, X. J. Am. Chem. Soc. 2018, 140, 16929.

(Li, L.; Fan, Y.) 